Revue d'histoire de l'Amérique française

MA REVUE D.HISTOIRE DE L'AMÉRIQUE FRANÇAISE

\title{
La pratique de l'histoire publique et la commémoration contemporaine : aperçu et enjeux
}

\section{Alexandra Mosquin, Danielle Hamelin et Catherine Cournoyer}

Volume 57, numéro 1, été 2003

L'histoire « publique » : un enjeu pour l'histoire

URI : https://id.erudit.org/iderudit/008355ar

DOI : https://doi.org/10.7202/008355ar

Aller au sommaire du numéro

Éditeur(s)

Institut d'histoire de l'Amérique française

ISSN

0035-2357 (imprimé)

1492-1383 (numérique)

Découvrir la revue

Citer cet article

Mosquin, A., Hamelin, D. \& Cournoyer, C. (2003). La pratique de l'histoire publique et la commémoration contemporaine : aperçu et enjeux. Revue

d'histoire de l'Amérique française, 57(1), 79-89. https://doi.org/10.7202/008355ar d'utilisation que vous pouvez consulter en ligne. 


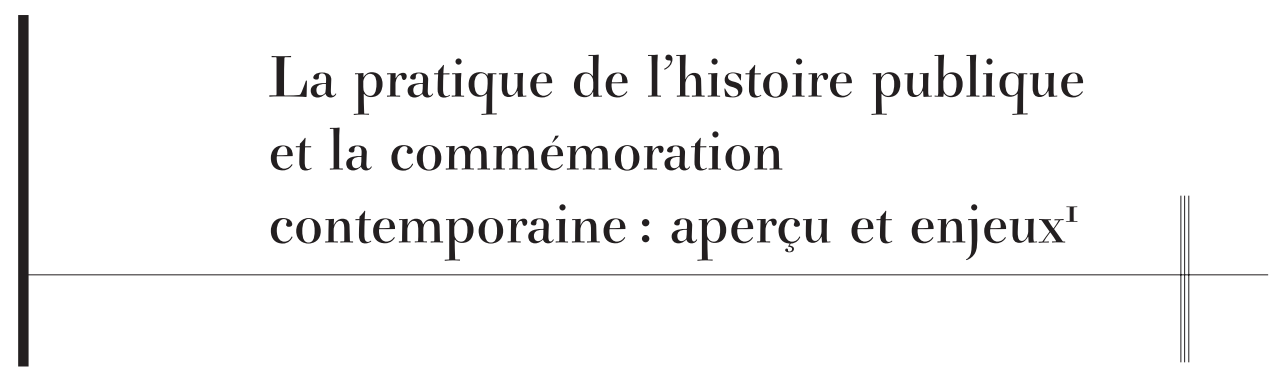

\author{
ALEXANDRA MOSQUIN \\ DANIELLE HAMELIN \\ CATHERINE COURNOYER \\ Parcs Canada
}

\begin{abstract}
A u cours des quinze DeRnières anNÉEs, un intérêt croissant pour la Apratique de l'histoire publique a marqué le milieu universitaire canadien. C'est du moins ce que laissent entendre la mise en place de cours et de programmes spécialisés en histoire publique, tout comme la production croissante d'articles et de monographies portant sur la commémoration historique et la conservation du patrimoine. Malgré cet engouement pour l'histoire publique, l'historien du domaine appartient à une espèce méconnue de la communauté historienne. Il participe pourtant à des colloques universitaires, publie à l'occasion dans des revues scientifiques et populaires et est souvent détenteur d'une maîtrise ou d'un doctorat. L'historien public évolue cependant dans un milieu bien différent du secteur universitaire puisque son travail est destiné à l'ensemble de la population. Par le fait même, il est souvent tiraillé entre la méthodologie et la connaissance historiographique acquises au cours de sa formation universitaire et les exigences des médias de l'histoire publique tels les expositions, les plaques commémoratives et les lieux historiques.

1. Nous tenons à remercier Geneviève Charrois, historienne de l'architecture à Parcs Canada, pour ses commentaires.
\end{abstract}


À titre d'historiennes travaillant au sein de la Direction des lieux historiques nationaux de Parcs Canada depuis quelques années, nous avons été confrontées à divers débats qui déchirent parfois la communauté multidisciplinaire à la base de la pratique de l'histoire publique. Sortant des sentiers battus du monde universitaire, la pratique de l'histoire publique dans le cadre d'un programme de commémoration soulève des questions difficiles : comment définit-on l'importance historique? Comment tranche-t-on quant à l'importance locale ou nationale d'un sujet historique? Qu'est-ce qui fait d'un site un lieu historique significatif? Ou encore, quelle est la place de l'histoire populaire et de la mémoire collective dans la pratique de l'histoire publique? Dans les pages qui suivent, nous aborderons certaines de ces questions afin de donner au lecteur un bref aperçu des enjeux auxquels fait face l'historien travaillant dans le cadre d'un programme de commémoration historique.

Lorsqu'il se joint à un organisme d'histoire publique, l'historien est confronté à une pratique de l'histoire différente de celle en vogue dans le milieu universitaire. Dans le cas de la Direction des lieux historiques nationaux du Canada, son travail est principalement destiné au soutien du programme de commémoration de la Commission des lieux et monuments historiques du Canada (CLMHC) ${ }^{2}$. Formée principalement d'historiens, de spécialistes de l'architecture, de géographes et d'experts en patrimoine, la CLMHC est le comité chargé de conseiller la ministre du Patrimoine canadien dans le choix de personnages, lieux et événements d'importance historique nationale. Ces commémorations mènent ensuite à l'érection d'une plaque de bronze bilingue, parfois à la conclusion d'une entente de partage des frais avec un tiers en vue de préserver ou d'interpréter un lieu ou, plus rarement, quand il s'agit d'un

2. En 1919, le gouvernement canadien s'engagea à préserver et à aménager divers lieux d'importance historique par la création de la Commission des lieux et monuments historiques du Canada. Cet engagement découlait de divers facteurs. D'abord, il y eut la montée d'un mouvement de conservation principalement constitué d'associations locales dès la fin du $\mathrm{xIX}^{\mathrm{e}}$ siècle. De plus, le ministère de l'Intérieur - et plus particulièrement le Commissaire aux parcs nationaux, J. B. Harkin -, envisageait la formation de parcs historiques afin d'étendre à l'ensemble du Canada l'entreprise des parcs nationaux commencée en 1885. Permettant d'assurer la conservation et l'agrément de propriétés devenues obsolètes, telles les fortifications et les postes de traite, ce dessein prit forme par l'acquisition de Fort Howe, au Nouveau-Brunswick, en 1914 et de Fort Anne, en Nouvelle-Écosse, en 1917. Face aux multiples possibilités de parc historique, reflétées par les pressions exercées par les diverses associations de conservation, le ministère avait besoin d'un organisme doté d'une solide expertise pour faire le tri, d'où la formation de la Commission des lieux et monuments historiques. C. J. Taylor, Negociating the Past: The Making of Canada's Historic Parks and Sites (Montréal, McGill-Queen's University Press, 1990), 3-60. 
site ou d'un événement d'une rareté ou d'une importance exceptionnelles, à l'acquisition et à la mise en valeur d'un lieu historique national majeur. L'historien qui travaille au soutien du programme de commémoration rédige les rapports sur lesquels la CLMHC base en partie ses recommandations de désignation. Il n'a donc pas pour fonction principale de contribuer à l'avancement du savoir en faisant une contribution originale, mais plutôt celle d'offrir une synthèse de la connaissance sur un sujet proposé dans le but d'établir le bien-fondé d'une commémoration. Ces rapports se fondent généralement sur l'historiographie établie et offrent une analyse à partir de la question de base du processus de commémoration : le sujet proposé «[...] a[t-il] eu une incidence marquante sur l'histoire du Canada ou constitue[t-il] une illustration ou un exemple important de l'histoire humaine du Canada ${ }^{3}$ ?». En d'autres mots, l'historien établit un pont entre le corpus de connaissances sur un sujet proposé et la commémoration de celui-ci en procédant à une analyse de l'importance historique.

Mais, précisément, comment juge-t-on l'importance historique nationale d'un sujet? Cet exercice visant à établir si un sujet est exceptionnel ou, encore, représentatif d'une facette importante de l'histoire canadienne, exige une approche intellectuelle étrangère à tout historien formé dans le milieu universitaire où règne le relativisme. Dans la communauté historienne en général, un sujet historique est intéressant en soi et toutes les facettes doivent être explorées pour le développement d'une connaissance plus complète du passé. Les historiens de la Direction des lieux historiques nationaux sont plutôt confrontés à une hiérarchisation des sujets historiques, c'est-à-dire qu'ils doivent situer les sujets dans un contexte comparatif national afin de définir s'ils sont ou non d'importance, ce qui nécessite un grand discernement et un esprit de synthèse développé. Il s'agit donc de trier les informations disponibles pour ne s'attarder qu'aux aspects dits nationaux, ce qui oblige à délaisser des facettes historiquement intéressantes, mais d'envergure locale. Dans le cas de la commémoration d'un individu, il faut établir si ses accomplissements sont de valeur supérieure ou égale à ceux des commémorations passées et surtout, si ses accomplissements ont une portée nationale. Un

3. Commission des lieux et monuments historiques du Canada, Critères - Lignes directrices générales - Lignes directrices particulières pour l'évaluation des sujets d'importance historique nationale (Gatineau, Parcs Canada, automne 2002), 1. Voir également le site Internet de Parcs Canada, $\dot{A}$ propos des critères, des lignes directrices générales et des lignes directrices particulières, http:// www.pc.gc.ca/clmhc-hsmbc/crit/index_f.asp. 
événement ne peut être considéré d'importance historique nationale que s'il constitue un tournant, un jalon ou une occurrence ayant des répercussions durables sur le cours de l'histoire canadienne. Finalement, l'analyse d'un lieu nécessite l'identification de ses associations avec un aspect important de l'histoire du Canada.

La notion d'importance historique a toujours été difficile à définir. À l'instar de l'historiographie canadienne, elle est en constante évolution. Ainsi, la nature même des sujets portés à l'attention de la CLMHC s'est diversifiée au fil des ans. Au moment de sa création, en 1919, certains membres de la CLMHC avaient une définition quasi constitutionnaliste de ce qui était d'importance nationale, une approche limitée aux domaines de compétence fédérale tels qu'ils sont définis par la section 91 de l'Acte de l'Amérique du Nord britannique 4 . Ils voyaient comme d'importance les personnages, les lieux et les événements qui témoignaient de l'érection de la nation canadienne, à savoir les forts, les emplacements de batailles, les postes de traite et autres hauts-lieux associés aux explorations et à l'expansion du Dominion. Cette notion d'importance reléguait à l'histoire locale plusieurs lieux aujourd'hui reconnus comme d'importance historique nationale. Par exemple, la Commission rejeta la commémoration du Manoir Papineau en 1920, jugeant que ce lieu était " only of local interest and not of any great value as a Historic Site, since no historic event took place there ${ }^{5}$ ». Un lieu historique national administré par Parcs Canada, le Manoir Papineau, fut finalement désigné en 1986 en raison des valeurs architecturales de la résidence, du lien avec l'histoire du système seigneurial au Québec et, aussi, du «reflet que constitue ce bâtiment de la personnalité de Louis-Joseph Papineau ${ }^{6}$ », personnage

4. Par exemple, lorsque le phare Sambro, premier phare construit au Canada, fut porté à l'attention de la Commission, le membre de la Colombie-Britannique, le juge Howay, justifia la désignation de ce site en affirmant que les «lighthouses are a subject which under the British North America Act fall within the purview of the Dominion Government, and are from that point of view matters of national importance». Correspondance entre Howay et Harkin, 22 octobre 1924. Archives nationales du Canada, RG 84, A-2-a, vol. 1200, HS6-25, pt. 1, T-13529. Ce type d'argument fut également utilisé pour justifier la désignation de sujets liés aux Autochtones comme les routes de portage et les champs de bataille dits traditionnels, encore une fois la Commission affirmant que "Indian affairs being under Dominion jurisdiction", elles étaient d'importance nationale. Correspondance entre Coyne et Harkin, 2 mars 1922. Archives nationales du Canada, RG 84, A-2-a, vol. 1329, HS9, pt. 3, T-14092.

5. Procès-verbaux de la Commission des lieux et monuments historiques du Canada, 20 mars 1920.

6. Procès-verbaux de la Commission des lieux et monuments historiques du Canada, 16 au 18 juin 1986. 
reconnu d'importance historique nationale en 1937. En élargissant de la sorte la notion d'importance historique pour inclure d'autres facettes importantes du passé, la Commission a ouvert la porte à un nombre illimité et à une grande diversité de possibilités de commémoration.

Un deuxième enjeu est l'analyse de l'importance historique d'un lieu, qu'il s'agisse d'un édifice, d'un complexe, d'un arrondissement ou d'un paysage culturel. Sujet à débat, cette analyse soulève une question fondamentale: est-ce que l'importance d'un lieu réside dans les qualités esthétiques, dans son association avec des événements historiques, ou encore, dans la valeur symbolique qu'il a aux yeux d'une communauté donnée? Plusieurs édifices ont été reconnus comme des lieux historiques nationaux en raison de l'excellence de leur design et du savoirfaire démontré par les artisans et bâtisseurs; d'autres l'ont été pour leur histoire ou encore, pour une combinaison de raisons architecturales et historiques. Bien que des lieux puissent être commémorés pour leur histoire, certains concepts clés du mouvement de conservation viennent aujourd'hui circonscrire la notion «d'association historique». Tout lieu doit passer le test de l'intégrité, de l'authenticité physique, un simple lien idéel ne pouvant suffire à une commémoration. Il faut dire qu'il n'en a pas toujours été ainsi. Au cours des années 1920 et 1930, par exemple, la CLMHC accordait surtout de la valeur à un lieu si un événement ou un personnage important y était associé, portant alors peu d'intérêt à l'intégrité physique des ressources matérielles d'un site. C'est du moins ce que laisse entendre Benjamin Sulte, membre de la Commission pour la province de Québec, dans une lettre adressée à J. B. Harkin, en 1919, au sujet de la désignation des Forges du Saint-Maurice: "All that can be done in our days is to clear away the heap of stones, in order to reach the foundation walls and plant a sign in the centre of the square thus uncovered ${ }^{7}$. " À cette époque, la CLMHC était davantage intéressée par la commémoration historique que par la conservation et ne jugeait pas nécessaire la présence de vestiges physiques pour commémorer un lieu ${ }^{8}$.

7. Cité dans C. J. Taylor, Negociating the Past..., op. cit., 51.

8. Cette approche fut fortement remise en question dans les années 1940, le président de la Commission d'alors cherchant à mettre davantage l'accent sur la conservation de lieux à caractère historique plutôt que de simplement ériger des plaques commémoratives. En 1951, la Commission Massey abonda dans ce sens en émettant l'avis que davantage d'efforts devraient être consacrés à la conservation de l'environnement bâti de valeur historique. De même, la Loi sur les lieux et monuments historiques de 1953, telle qu’elle a été amendée en 1955, définit les lieux historiques comme : «Emplacement, bâtiment ou autre endroit d'intérêt ou d'importance historique nationale, y compris les bâtiments ou ouvrages qui sont d'intérêt national en raison de 
Le tournant vers une conservation axée sur les concepts d'intégrité physique et d'esthétique qui a marqué la seconde moitié $d u x^{e}$ siècle a, par ailleurs, rendu difficile la reconnaissance de divers lieux importants du passé en les confrontant à une grille d'analyse privilégiant l'environnement bâti formel aux associations et messages historiques. Le problème de commémorer un patrimoine physiquement modeste, voire éphémère ou même intangible, tels les lieux sacrés et spirituels de certaines communautés autochtones, ou de commémorer des mouvements et des organisations qui ont souvent évolué dans des maisons et autres bâtiments moins prestigieux que les grandes institutions avec colonnes grecques, tourelles et clochers érigées par les élites, ne cesse d'être préoccupant. Par exemple, peu de lieux historiques nationaux relatent l'histoire des mouvements ouvriers au Canada. Commémorer l'histoire nationale des groupes et individus longtemps marginalisés de la grande narration historique tout comme les bâtiments souvent plus simples et modestes qui leur sont associés exige de se détacher des notions de style et d'esthétique. Pour contrer ces difficultés, il s'agit, entre autres, de mettre l'accent sur la capacité d'un lieu de faire passer le message, de véhiculer son histoire. La tâche de l'historien est dès lors d'identifier les liens entre les caractéristiques physiques d'un bâtiment et ses valeurs historiques. Des constructions aussi modestes que des cabanes en bois rond et des huttes de terre peuvent être considérées comme lieu historique potentiel tant et aussi longtemps que leur état physique et leur emplacement peuvent évoquer un épisode particulier de l'histoire. Mais si les matériaux, les traces des techniques de construction de l'époque et le caractère originel de l'emplacement ont été détruits, est-ce qu'un bâtiment donné possède toujours un intérêt historique? Ou encore, si un

leur âge ou de leur architecture.» Cette allusion aux styles architecturaux, qui coïncidait avec la montée de l'étude de l'histoire de l'architecture au Canada, enclencha une mouvance vers la conservation de bâtiments et autres structures non seulement pour leur valeur historique, mais également esthétique. Les décennies qui suivirent furent marquées, d’une part, par la commémoration d'édifices représentatifs d'un certain type de bâtiment, tels que des hôtels de ville, des palais de justice, des écoles ou des églises et, d'autre part, par la commémoration de beaux exemples de styles architecturaux, allant du néo-gothique au néo-Queen Anne. Dès lors, l'accent fut mis sur l'intégrité physique d'un lieu, l'association historique ne pouvant seule mener à une désignation d'importance nationale. C. J. Taylor, Negociating the Past..., op. cit., 128; voir également Shannon Ricketts, "The Built Environment: Changing Direction in Federal Commemoration", Rapport au feuilleton pour la Commission des lieux et monuments historiques du Canada, 1994-50, juin 1994.

9. Brian Graham, «Heritage as Knowledge: Capital of Culture?», Urban Studies, 39,5-6 (2002): 1005 . 
bâtiment a été retiré de son emplacement originel, c'est-à-dire délogé de ses fondations et installé dans un autre endroit, conserve-t-il un intérêt historique? Face à ces problématiques, il faut souvent procéder cas par cas $^{10}$.

Une autre matière à débat est le type d'histoire qui doit ou devrait être commémoré. Quelle place doit-on accorder aux facettes controversées, voire douloureuses, dans un programme qui se veut une célébration de notre passé commun? Si, à ses débuts, les sujets commémorés par la Commission laissaient transparaître un désir d'édifier une identité nationale reposant sur des mythes fondateurs, la réalité est tout autre aujourd'hui. Dès 1955, la Commission recommanda que la déportation des Acadiens soit reconnue comme un événement d'importance historique nationale. Autrefois rare, la désignation de facettes moins glorieuses du passé est de plus en plus courante, ce qui est en partie attribuable à une évolution de la définition de l'importance historique à la suite du développement des connaissances par la communauté scientifique des historiens. En effet, l'évolution de l'historiographie canadienne témoigne des changements de perception qui ont marqué la notion d'importance historique, la démocratisation des études universitaires dès les années 1960 ayant engendré la montée de l'histoire sociale, celle-ci levant le voile sur le passé des marginalisés de l'histoire tels les Autochtones, les communautés ethniques et les femmes, d'ailleurs les trois priorités actuelles du programme de commémoration. Ainsi, en 1977, la Commission recommandait la désignation des travailleurs chinois du chemin de fer Canadien Pacifique, en 1984, l'internement des Japonais lors de la Seconde Guerre mondiale et, en 1996, l'emplacement de ce qui fut une des plus importantes communautés noires du Canada détruite au nom de la réhabilitation urbaine: Africville, à Halifax, en Nouvelle-Écosse. Du passé glorieux d'un Canada fort et vertueux, où seuls les événements soi-disant fondateurs de la nation tels la traite des fourrures, les campagnes militaires, les forts et quelques hauts faits méritaient une place au panthéon du passé, la Commission a évolué, au cours de la seconde moitié $d u x^{e}$ siècle, vers la commémoration d'un passé pluriel.

Cette ouverture aux épisodes plus douloureux de l'histoire canadienne soulève cependant une question fondamentale dans le domaine de

10. Si l'emplacement originel est un élément historique clé, il est entendu que le bâtiment déménagé perd son intérêt patrimonial. 
l'histoire publique: qui détermine la valeur historique, la notion d'importance nationale? L'histoire publique est par définition écrite pour l'ensemble de la population, ce qui sous-entend une interaction entre le passé, l'historien et le public. Alors que le programme de commémoration tente de plus en plus d'inclure l'histoire des groupes autrefois à la marge du grand récit national, il apparaît que les communautés concernées doivent être incluses dans cette grande aventure. Dès lors, des questions méthodologiques se posent: quelle place doit occuper la perspective du public ou d'une communauté donnée dans la rédaction d'un rapport? Confronté au devoir de consulter les communautés, l'historien qui idéalement agit en expert détaché devient un filtre entre la communauté et la CLMHC, distillant en quelque sorte les différentes couches de signification retrouvées dans la communauté pour les insérer dans le contexte historique et comparatif.

La diversité des possibilités et le nombre croissant de commémorations ont entraîné la mise en place d'un cadre politique visant à circonscrire l'exercice commémoratif. L'historien de Parcs Canada évolue donc à l'intérieur d'une gamme variée de lois, critères et lignes directrices et contribue même régulièrement au développement de ce cadre politique. Reposant soit sur une analyse des précédents de la CLMHC et des commémorations passées ou sur une étude exhaustive d'un sujet afin d'en dégager le contexte national, cet ensemble de règles sert à assurer la fonctionnalité, la logique et la rigueur du processus de commémoration. En fait, ce cadre facilite le traitement des demandes de désignation en provenance du public, des organismes patrimoniaux et des administrations provinciales et municipales. En raison des pressions de divers individus et organismes désireux d'obtenir une désignation afin d'assurer la pérennité de leurs propriétés, et dans un contexte gouvernemental où règnent les exigences d'imputabilité et de diligence, ce cadre politique est essentiel. Bref, l'historien qui travaille dans un cadre bureaucratique est une sorte de bête mutante puisqu'il est amené à adopter des automatismes propres aux juristes et aux politologues.

Ce cadre politique cherche à prévenir la commémoration de sujets dont l'importance historique ne serait que temporelle ou dérivée des préoccupations idéologiques du présent. Contrairement à la croyance générale, le programme de commémoration n'est pas une entreprise patrimoniale au sens strict du terme, soit un organisme qui accorde de l'importance à un sujet uniquement à la lumière de sa signification dans le présent et des bénéfices économiques, politiques et culturels pouvant 
en découler ${ }^{11}$, mais surtout une initiative de diffusion de l'histoire. Parmi les critères de base se trouvent les règles de temps, d'où l'exigence d'un recul de quarante ans pour considérer la commémoration de tout événement historique. Cette règle vise à assurer que le sujet considéré bénéficie d'une connaissance suffisamment développée pour en jauger l'importance, ce qui suppose que les historiens universitaires ont d'ores et déjà fait leur travail de recherches et d'analyse. La même logique s'applique à la règle voulant qu'un individu soit considéré pour une désignation d'importance historique seulement vingt-cinq ans après son décès. Les édifices ou les ensembles de bâtiments doivent, de leur côté, avoir été complétés avant 1975 pour être considérés.

Notons également que la nature de notre travail laisse peu de place à l'idiosyncrasie ou à l'adoption d'une perspective donnée dans le traitement des sujets lors de la rédaction des rapports à la CLMHC. D'abord, ce rapport est écrit dans le but de mener à une "raison de désignation", soit à la rédaction d'un court énoncé formulé par la CLMHC et spécifiant la nature de l'importance historique nationale du sujet proposé. Cette raison de désignation mène pour sa part au produit final: une plaque de bronze comportant une inscription de 600 caractères dans chacune des langues officielles. Le rapport doit donc permettre à la Commission de prendre une position éclairée sur le sujet proposé. Ici réside une des principales différences avec la pratique universitaire. Dans le domaine universitaire, un historien a le privilège d'adopter une perspective donnée sur son sujet d'études tant et aussi longtemps qu'il fait preuve d'honnêteté intellectuelle en exposant clairement les sources sur lesquelles repose son raisonnement. Ce privilège de la divergence n'est pas accordé à l'historien qui travaille dans le cadre d'un programme de commémoration dont l'un des objectifs est d'émettre une plaque brève véhiculant un message ayant la prétention de durer.

Loin de prétendre à l'idéal de la neutralité intellectuelle, disons que l'historien public doit concilier des points de vue divergents, voire chercher un compromis entre ceux-ci ou encore, trancher entre les différentes interprétations afin de répondre aux exigences des médias de la commémoration. Si l'histoire publique peut reconnaitre les multiples

11. "Heritage itself is conceptualized as the meanings attached in the present to the past and is regarded as a knowledge defined within social, political and cultural contexts ", B. Graham, "Heritage as Knowledge», op. cit., 1003. 
interprétation $\mathrm{s}^{12}$, elle ne vit pas très bien avec celles-ci au quotidien car, il faut bien le reconnaître, ces débats ne s'insèrent pas très bien sur une plaque de bronze, aussi grande soit-elle. Hélas! Nous n’en sommes pas encore aux plaques interactives!

Certains diront qu'il s'agit là de la plus grande faiblesse de la commémoration historique. En effet, certains spécialistes de la question patrimoniale reprochent à ce domaine de diffusion de l'histoire de ne pas offrir à son public les visions discordantes et les multiples interprétations qui cohabitent dans l'espace historiographique. Force est d'admettre que la commémoration est façonnée par son auditoire et ses médias ${ }^{13}$. Devant être intelligible pour l'homme de la rue et l'enfant d'âge scolaire, les plaques ne peuvent véhiculer toute la complexité de l'historiographie canadienne. Elles optent plutôt pour un narratif continu et cohérent, laissant généralement peu de place au doute et à la controverse. Il en résulte parfois une histoire aseptisée, emplie de compromis et de nondits, mais plus facile à assimiler. Brève, elle véhicule l'importance de certains sujets dans l'espoir de susciter l'intérêt du lecteur et, ainsi, contribuer à une meilleure connaissance du passé chez l'ensemble des citoyens.

Avec une population vieillissante qui s'intéresse de plus en plus à son histoire et une industrie touristique florissante qui gagne à mettre en valeur les lieux historiques, ces débats quant à l'importance historique et à ce qui constitue des lieux historiques devraient préoccuper toute personne intéressée par l'histoire qui est racontée au grand public. Les diverses plaques de bronze et lieux historiques qui parsèment le territoire canadien marquent l'importance de divers personnages, lieux et événements dans l'histoire, constituant des médias privilégiés, bien

12. Dans le cadre de l'interprétation faite aux lieux historiques nationaux, il y a place pour les discordantes visions de l'histoire. Ainsi, la politique de gestion des ressources culturelles de Parcs Canada stipule, à l'article 1.5.1: «L'ensemble de l'histoire est mis en valeur avec intégrité, c'est-à-dire qu'on fait valoir des points de vue contemporains divergents, des perspectives fondées sur le savoir traditionnel, ainsi que des interprétations plus récentes. Parcs Canada n’assume pas un rôle d'arbitre au regard de l'histoire humaine du Canada ». Parcs Canada, «Politique sur la gestion des ressources culturelles", Principes directeurs et politiques de gestion, 108.

13. «Au fil des ans, la communication de l'importance nationale des lieux historiques a été influencée par un certain nombre de facteurs: conformisme politique, consultation publique, interprétation destinée à satisfaire les demandes de la population, programmes de reconstitution de l'histoire». Christina Cameron, "Commemoration: A Moving Target», dans Thomas H. B. Symons, dir., Les lieux de mémoire: la commémoration du passé du Canada - Actes du symposium national tenu à l'occasion du $75^{\circ}$ anniversaire de la Commission des lieux et monuments historiques $d u$ Canada (Ottawa, la Société royale du Canada, 1997), 27-28. 
qu'imparfaits, pour rejoindre le public canadien et informer les touristes sur qui nous sommes comme peuple. Étant nous-mêmes des adeptes du tourisme de plaques et de lieux historiques, nous avons été en mesure d'apprécier la réaction des gens à la suite de la lecture de plaques ou de la visite de lieux historiques. Par le fait même, nous jugeons essentiel que les historiens du milieu universitaire, en raison de leur expertise, s'engagent dans le processus commémoratif afin que notre réseau de plaques et de lieux historiques reflète le mieux possible l'ensemble du passé. 